\title{
Impact of contact lens wear on NLRP3 gene expression: Implications for ocular frailty in middle-aged adults
}

\author{
Almudena Crooke ${ }^{\mathrm{a},{ }^{*}}$, Irene Martínez-Alberquilla ${ }^{\mathrm{b}}$, María García-Montero ${ }^{\mathrm{b}}$, \\ Laura Rico-del-Viejo $^{\mathrm{b}}$, Javier Ruiz-Alcocer ${ }^{\mathrm{b}}$, David Madrid-Costa ${ }^{\mathrm{b}}$ \\ ${ }^{\text {a }}$ Department of Biochemistry and Molecular Biology, Faculty of Optics and Optometry, Universidad Complutense de Madrid, Madrid, Spain \\ ${ }^{\mathrm{b}}$ Department of Optometry and Vision, Faculty of Optics and Optometry, Universidad Complutense de Madrid, Madrid, Spain
}

\section{A R T I C L E I N F O}

\section{Keywords:}

Inflammaging

Inflammasome

Mucin

Ectonucleotidase

Ocular frailty

\begin{abstract}
A B S T R A C T
The inflammatory process plays a crucial role in frailty syndrome, which can appear in middle age and is associated with a poor health outcome. Consequently, gerontologists recommend screening inflammatory biomarkers in middle-aged adults to detect frailty and, therefore, prevent chronic diseases and mortality. External factors could be a risk factor for frailty because they can generate and extend the inflammatory process. For these reasons, we analysed the effect of long-term contact lens wear on mRNA level of genes linked to inflammation (IL-6, NLRP3, NK1R, CD73, MUC16 and TRPV1 genes) in conjunctival cells of middle-aged individuals, by quantitative PCR. Middle-aged contact lens wearers presented a significant increase of NLRP3 and MUC16 mRNA level as well as a decrease of CD73 mRNA level, in comparison with non-contact lens wearers. Additionally, we checked for a potential correlation between these transcript levels and clinical changes of the participants' ocular surface. Unlike molecular analysis, clinical examination fails to detect inflammation in contact lens wearers. These data suggest that long-term contact lens wear could trigger an inflammatory response in middle age orchestrated by NLRP3 inflammasome and modulated by CD73 and MUC16 proteins. Further studies are needed to confirm our gene expression findings at the protein level as well as to investigate the potential role of longterm CL wear in the onset of ocular frailty.
\end{abstract}

Middle age is a crucial period in life in which the decline of the body's regulatory systems (ageing) begins (Lachman et al., 2015; Lehallier et al., 2019). Indeed, Lehallier and colleagues have demonstrated a wave of proteomic change in the fourth decade of life that reflects the biological relevance of this period (Lehallier et al., 2019). Furthermore, various studies have detected frailty syndrome in middle-aged adults in whom this syndrome is a poor prognostic factor (Hanlon et al., 2018; Prince et al., 2019). Consequently, authors recommend screening frailty biomarkers in middle-aged people to prevent adverse health outcomes (Hanlon et al., 2018; Prince et al., 2019). The most convincing frailty biomarkers are inflammation-related candidates (Prince et al., 2019). Indeed, inflammation is a common pathway of frailty and age-related diseases (Fulop et al., 2010).

External factors could be a risk factor for frailty because they can generate and extend the inflammatory process. Indeed, they are pivotal in age-related ocular disease onsets (Crooke et al., 2017). Villani and colleagues have recently applied the concept of ocular surface frailty to an unhealthy ocular surface predisposed to age-related dry eye disease (Villani et al., 2019). In this context, contact lens (CL) wear could be a risk factor for this specific type of frailty. In relation to this issue, some authors have demonstrated that CL wear triggers an inflammatory response in young adults (Lopez-de la Rosa et al., 2019; Saliman et al., 2020). Moreover, CL wear is considered a risk factor of dry eye disease (Kojima, 2018). For these reasons, we have investigated the potential inflammatory effect of long-term CL wear in middle age.

Twenty-six middle-aged individuals (age range: 42-65 years), 12 non-contact lens wearers (NCLW; $\mathrm{n}=12$; mean [SD] age: 53.8 [7.0] years; 8 females and 4 males) and 14 contact lens wearers (CLW; $n=14$; mean [SD] age: 53.9 [4.1] years; 9 females and 5 males) with a CL wearing routine of $8 \mathrm{~h}$ per day for at least 1 year were enrolled for this study. Individuals with active ocular allergy, ocular inflammatory diseases, refractive surgery or systemic medication were excluded. The study was approved by the ethics committee of the Hospital Clínico San Carlos and written informed consent was obtained from each

\footnotetext{
* Corresponding author. Department of Biochemistry and Molecular Biology Faculty of Optics and Optometry, Universidad Complutense de Madrid C/Arcos de Jalón, 118 28037, Madrid, Spain.

E-mail address: acrooke@ucm.es (A. Crooke).
} 
participant.

All subjects underwent a standard clinical examination of the ocular surface (Wolffsohn et al., 2017) to evaluate the gross impact of CL wear. The clinical protocol included evaluation of ocular irritation symptoms and assessment of the tear film stability and the ocular surface health status.

The Ocular Surface Disease Index (OSDI) questionnaire (Schiffman et al., 2000)was used to evaluate symptoms of ocular irritation. The formula used by Schiffman and colleagues (Schiffman et al., 2000) was applied to obtain the final OSDI score. The higher the score, the more severe the symptoms and the greater the disability.

The tear meniscus height (TMH) and first/average non-invasive tear film breakup time (NIKBUT first/avg) parameters were determined to evaluate tear film stability. The bulbar redness (BR) and corneal/ conjunctival integrity were determined to check the ocular surface health status.

The measure of TMH, NIKBUT first/avg and BR parameters were performed with the Keratograph 5M (Oculus GmbH, Wetzlar, Germany) device equipped with the modified tear film scanning function and obtained automatically from the K5M software following the manufacturer's directions. An assessment of corneal/conjunctival integrity was performed, 2 min after application of fluorescein sodium ophthalmic strips (FluoStrip, Contacare Ophthalmics and Diagnostics, Gujarat, India), with the Keratograph $5 \mathrm{M}$ (Oculus $\mathrm{GmbH}$ ) device and the $\mathrm{K} 5 \mathrm{M}$ blue-free barrier filter. Fluorescein corneal and conjunctival staining images were graded according to the Efron scale (Efron, 1998), ranging from 0 (normal) to 4 (severe). Conjunctival staining was divided into bulbar and tarsal sections.

After the clinical examination, and in order to evaluate the molecular impact of CL wear, a conjunctival specimen was obtained from each individual by impression cytology with the EYEPRIM device (OPIA Technologies, Paris, France). Conjunctival specimens were preserved in RNAlater solution (Thermo Fisher Scientific) overnight and, after removing the RNAlater, stored at $-80^{\circ} \mathrm{C}$ until total RNA isolation.

Total RNA was isolated from impression cytology specimens using RNeasy Mini Kit (Qiagen, Madrid, Spain), following the manufacturer's instructions. First-strand cDNA synthesis was performed from $22 \mu \mathrm{l}$ of total RNA, using High Capacity cDNA Reverse Transcription Kit and random hexamer primers (Thermo Fisher Scientific, Madrid, Spain). Quantitative PCR (qPCR) was performed in technical triplicates using cDNA, the Quantitect SYBR Green Kit (Qiagen) and gene-specific PCR primers for HPRT1 (QuantiTect Primer Assay QT00059066, Qiagen), IL6 (QuantiTect Primer Assay QT00083720, Qiagen), NLRP3 (QuantiTect Primer Assay QT00029771, Qiagen), NK1R (5'-CTACTACTCAACCACAGAGAC-3' /5'-CACAGATGTGGTACACTTTC-3', Merck, Madrid, Spain), CD73 (QuantiTect Primer Assay QT02451512, Qiagen), MUC16 (QuantiTect Primer Assay QT01192996, Qiagen) and TRPV1 (QuantiTect Primer Assay QT00046109, Qiagen), on an ABI Prism 7300 PCR System (Thermo Fisher Scientific). The thermal cycler program was 15 min at $95^{\circ} \mathrm{C}$, followed by 40 cycles of $15 \mathrm{~s}$ at $94^{\circ} \mathrm{C}, 30 \mathrm{~s}$ at $55^{\circ} \mathrm{C}$ and 34 $s$ at $72{ }^{\circ} \mathrm{C}$ (data collection step). The HPRT1 gene was used as an internal control to normalise mRNA relative expression, after its validation for qPCR. Validation of the internal control gene and qPCR data analysis were performed by the $2^{-\Delta \mathrm{Ct}}$ method (Schmittgen and Livak, 2008).

Statistical analysis was performed using IBM SPSS Statistics software version 25.0. (SPSS Inc., Chicago, IL, USA). Shapiro-Wilk and MannWhitney tests were used for statistical analysis of quantitative clinical (OSDI, TMH, NIKBUT first, NIKBUT avg and BR) and molecular (gene expression data of HPRT1, IL-6, NLRP3, NK1R, CD73, MUC16 and TRPV1) data. The chi-square test was used for statistical analysis of qualitative clinical data (corneal and conjunctival staining). All the values (clinical and molecular) are expressed as mean \pm SD and median. A statistical significance of $95 \%$ was established $(\mathrm{p}<0.05)$ in all the tests. Correlations among molecular and clinical variables were assessed through Kendall's Tau-b ( $\tau \mathrm{b}$ ) coefficient. The correlations were considered significant if $\mathrm{p}<0.05$.
To examine the mRNA level of inflammatory genes in conjunctival cells of NCLW and CLW by qPCR, we chose the HPRT1 gene as an internal control because its expression was unaffected by CL wear ( $p=$ 0.43) (Table 1). The levels of IL6, NK1R and TRPV1 mRNAs were comparable between CLW and NCLW ( $p>0.05$ ) (Table 1). Conversely, CLW showed a marked overexpression of NLRP3 and MUC16 genes (median 2.6-fold and 3.5-fold higher, respectively, than NCLW; $p<$ 0.05) (Fig. 1A-B; Table 1). Likewise, we observed a significant downregulation of CD73 gene in CLW (median 6.4-fold lower than NCLW; $p<$ 0.05) (Fig. 1C; Table 1).

Concurrently, we checked if CL wear alters the ocular surface of middle-aged adults at the clinical level (Table 1). Clinical parameters levels were comparable between CLW and NCLW ( $p>0.05)$ (Table 1).

Finally, we also studied the possible correlation between NLRP3, MUC16 and CD73 mRNA level and clinical parameters. We found only a significant negative correlation between tarsal conjunctiva staining and MUC16 mRNA level ( $\tau \mathrm{b}=-0.62 ; p=0.04)$.

This study shows, for the first time, the effect of CL wear on conjunctival NLRP3 and CD73 gene expression. Our results demonstrate a higher expression of the NLRP3 gene in CLW than NCLW. The NLRP3 inflammasome is a major contributor to the chronic low-grade inflammation that characterises frailty and age-related conditions including eye diseases (Fulop et al., 2010; Lim et al., 2020). Indeed, Niu and colleagues have proved the upregulation of NLRP3 mRNA and protein in ocular surface cells of dry eye patients (Niu et al., 2015). Furthermore, other authors have demonstrated that frail middle-aged adults present a race-specific alteration of IL1- $\beta$ mRNA (an effector of NLRP3 inflammasome) in blood cells (Prince et al., 2019).

Additionally, we have demonstrated that CLW present a lower CD73 mRNA level than NCLW. CD73 is an ectoenzyme that, along with the CD39 nucleotidase, degrades the extracellular inflammatory nucleotide adenosine triphosphate (ATP) to adenosine, a potent endogenous antiinflammatory agent (Hasko and Cronstein, 2013). In fact, ATP is a co-activator of NLRP3 inflammasome (Stagg and Smyth, 2010). Therefore, this result suggests that long-term CLW could be more vulnerable to the inflammatory process, due to the imbalance between the levels of

Table 1

Molecular and clinical parameters of the study groups.

\begin{tabular}{llll}
\hline & NCLW & CLW & p-value \\
\hline Molecular parameters & & & \\
HPRT1 & $1.09 \pm 0.46$ & $1.10 \pm 0.94$ & 0.43 \\
IL6 & $0.07 \pm 0.10$ & $0.11 \pm 0.16$ & 0.35 \\
NLRP3 & $0.01 \pm 0.01$ & $0.05 \pm 0.08$ & $\mathbf{0 . 0 2}$ \\
NK1R & $0.00 \pm 0.00$ & $0.00 \pm 0.01$ & 0.46 \\
CD73 & $1.72 \pm 0.85$ & $0.86 \pm 1.08$ & $\mathbf{0 . 0 2}$ \\
MUC16 & $0.50 \pm 0.28$ & $1.78 \pm 1.52$ & $\mathbf{0 . 0 4}$ \\
TRPV1 & $0.24 \pm 0.08$ & $0.29 \pm 0.20$ & 0.71 \\
Clinical parameters & & & \\
TMH (mm) & $0.30 \pm 0.16$ & $0.31 \pm 0.07$ & 0.50 \\
BR & $1.40 \pm 0.36$ & $1.33 \pm 0.30$ & 0.50 \\
NIKBUT first (sec) & $10.63 \pm 6.86$ & $10.01 \pm 5.59$ & 0.98 \\
NIKBUT avg (sec) & $13.73 \pm 6.48$ & $12.98 \pm 5.08$ & 0.65 \\
OSDI & $15.94 \pm 9.06$ & $13.19 \pm 9.70$ & 0.46 \\
Corneal staining (Efron scale) & $0.27 \pm 0.65$ & $0.00 \pm 0.00$ & 0.20 \\
SBC staining (Efron scale) & $0.00 \pm 0.00$ & $0.08 \pm 0.28$ & 1.00 \\
IBC staining (Efron scale) & $0.09 \pm 0.30$ & $0.08 \pm 0.28$ & 1.00 \\
NBC staining (Efron scale) & $0.73 \pm 0.79$ & $0.69 \pm 0.75$ & 1.00 \\
TBC staining (Efron scale) & $0.45 \pm 0.52$ & $0.23 \pm 0.60$ & 0.06 \\
TC staining (Efron scale) & $0.18 \pm 0.40$ & $0.15 \pm 0.38$ & 1.00 \\
\hline
\end{tabular}

Note: Molecular data are the mean \pm standard deviations (SD) of mRNA fold change. Clinical data are presented as the mean $\pm \mathrm{SD}$. * indicates statistically significant differences $(p<0.05)$ between NCLW and CLW. NCLW: non-contact lens wearers; CLW: contact lens wearers; TMH: tear meniscus height; BR: bulbar redness; NIKBUT first: non-invasive first tear film breakup time; NIKBUT avg: average time of all tear film break-up incidents; OSDI: Ocular Surface Disease Index questionnaire; SBC: superior bulbar conjunctiva; IBC: inferior bulbar conjunctiva; NBC: nasal bulbar conjunctiva; TBC: temporal bulbar conjunctiva; TC: tarsal conjunctiva. 

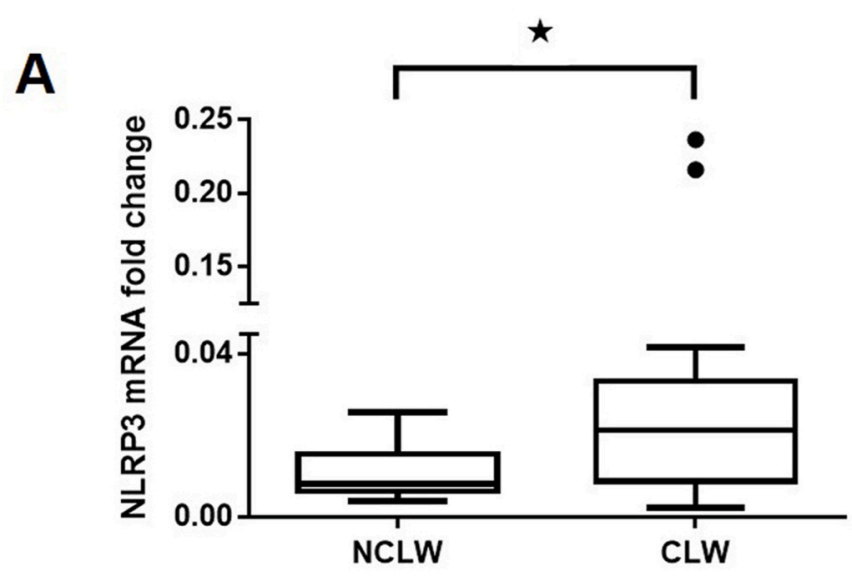

B
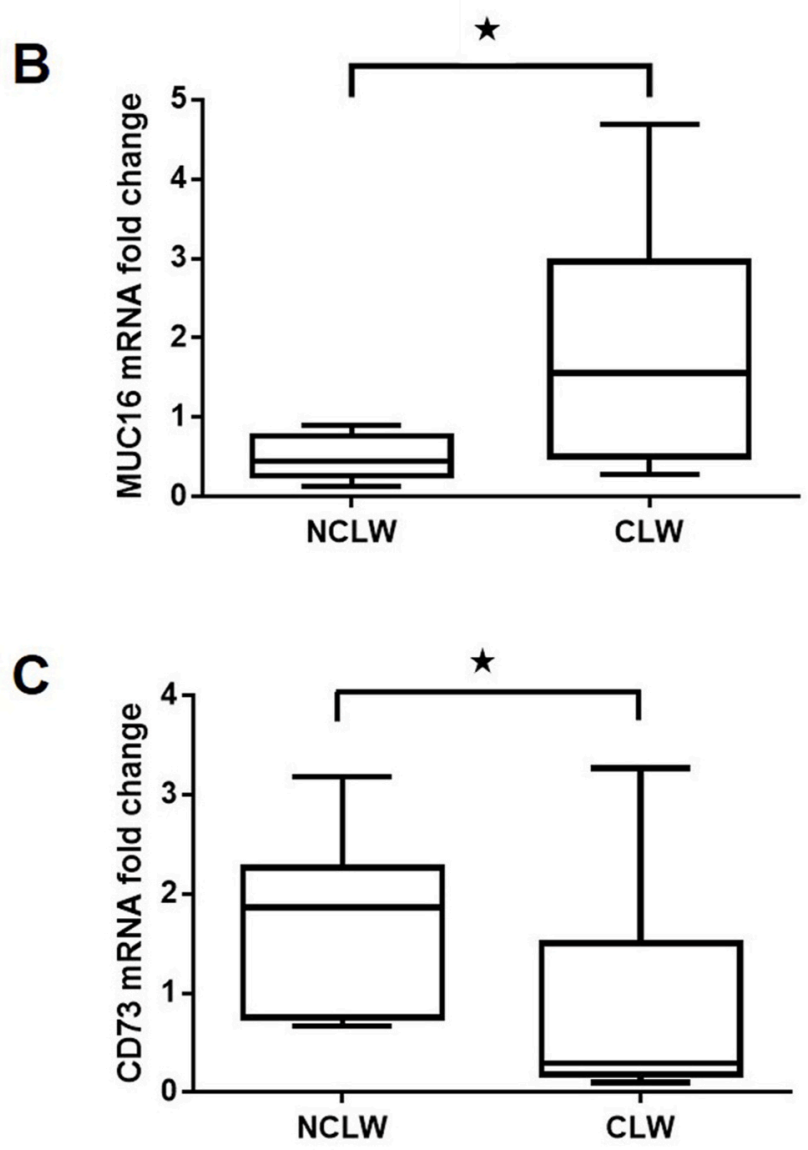

Fig. 1. Impact of contact lens wear on NLRP3 (A), MUC16 (B) and CD73 (C) gene expression in conjunctival cells of middle-aged adults. Gene expression level data for each gene and sample were normalised to HRPT1 signal (internal control). The horizontal line inside the box represents the median value, the lower and upper box lines represent the limits of the interquartile range (25th and 75th percentiles, respectively). Whiskers represent extreme values and dots depict outlier values. ${ }^{*}(p<0.05)$ indicates statistically significant differences between non-contact lens wearers and contact lens wearers. The $p$ values were determined by Mann-Whitney test.

ATP and adenosine, when they reach middle age.

Unlike the genomic effect on CD73, we have observed that CL wear induces MUC16 gene expression. The effect of CL wear on mucin expression has been previously analysed but the results are inconclusive because the studies were conducted without a clearly stated participant age distribution or with age-unmatched between compared groups
(Ramamoorthy and Nichols, 2008). MUC16 is a membrane-bound mucin essential to maintain tear film quality and tear film stability, therefore changes in its expression promote ocular surface diseases (Baudouin et al., 2019). In fact, Gipson and colleagues found that postmenopausal women with dry eye disease overexpress MUC16 (Gipson et al., 2011). These authors suggest that this effect is a mechanism to compensate for disease-associated inflammation (Gipson et al., 2011). In this context, some authors have demonstrated that ATP activates NLRP3 inflammasome to induce mucins production in airway epithelial cells (Kim et al., 2018). Furthermore, Paulsen and colleagues found that IL1- $\beta$ upregulates conjunctival MUC16 mRNA expression (Paulsen et al., 2008). Reciprocally, mucin levels may lead to tear hyperosmolarity that can activate NLRP3 inflammasome (Zheng et al., 2015). All these facts support our results; we have found that long-term CL wear downregulates the CD73 gene (and so the level of ATP can be increased) and upregulates the NLRP3 and MUC16 genes in conjunctival cells of middle-aged wearers.

We have also assessed a potential correlation between these molecular changes and clinical damage. From our results and those of other authors (Chao et al., 2017), it seems that clinical parameters may fail to detect early stages of inflammation in CLW. In this context, we have observed that MUC 16 mRNA level of CLW is inversely proportional to tarsal conjunctiva staining. This result suggests that the expression of MUC16 in ocular surface cells avoids staining. Indeed, Blalock and colleagues have demonstrated this barrier role of MUC16 (Blalock et al., 2008). Consequently, we believe that ocular surface inflammation should be analysed using molecular techniques to detect it early.

All these preliminary results allow us to hypothesise that the longterm use of CL could be a risk factor of ocular surface frailty in middle-aged individuals. Given that frailty predisposes individuals to age-related diseases (Fulop et al., 2010; Hanlon et al., 2018) and that there are more than 140 million CLW worldwide (Stapleton et al., 2007), this is an essential issue. Indeed, Chen and colleagues have observed that a high rate of CLW developed glaucoma and retinal diseases during their CL wearing experience (Chen et al., 2020).

In summary, our gene expression findings suggest that long-term CL wear could trigger an inflammatory response orchestrated by NLRP3 inflammasome and modulated by CD73 and MUC16 proteins in middle age. Further studies are needed to confirm our findings at the protein level as well as to investigate the potential role of long-term CL wear in the onset of ocular frailty.

\section{Funding}

This work was supported by the European Union's Horizon 2020 research and innovation program under the Marie Sklodowska-Curie Actions (grant number 642760).

\section{Declaration of interest}

None.

\section{Acknowledgments}

We thank Pedro Cuesta (Data Processing Center, Universidad Complutense de Madrid, Spain) for help with statistical analyses.

\section{References}

Baudouin, C., Rolando, M., Benitez Del Castillo, J.M., Messmer, E.M., Figueiredo, F.C., Irkec, M., Van Setten, G., Labetoulle, M., 2019. Reconsidering the central role of mucins in dry eye and ocular surface diseases. Prog. Retin. Eye Res. 71, 68-87.

Blalock, T.D., Spurr-Michaud, S.J., Tisdale, A.S., Gipson, I.K., 2008. Release of membrane-associated mucins from ocular surface epithelia. Invest. Ophthalmol. Vis. Sci. 49, 1864-1871.

Chao, C., Richdale, K., Jalbert, I., Doung, K., Gokhale, M., 2017. Non-invasive objective and contemporary methods for measuring ocular surface inflammation in soft 
contact lens wearers - a review. Contact Lens Anterior Eye : J. Br. Contact Lens Assoc. 40, 273-282.

Chen, E.Y., Myung Lee, E., Loc-Nguyen, A., Frank, L.A., Parsons Malloy, J., Weissman, B. A., 2020. Value of routine evaluation in asymptomatic soft contact lens wearers. Contact Lens Anterior Eye: J. Br. Contact Lens Assoc. 43, 484-488.

Crooke, A., Huete-Toral, F., Colligris, B., Pintor, J., 2017. The role and therapeutic potential of melatonin in age-related ocular diseases. J. Pineal Res. 63.

Efron, N., 1998. Grading scales for contact lens complications. Ophthalmic Physiol. Optic. 18, 182-186.

Fulop, T., Larbi, A., Witkowski, J.M., McElhaney, J., Loeb, M., Mitnitski, A., Pawelec, G., 2010. Aging, frailty and age-related diseases. Biogerontology 11, 547-563.

Gipson, I.K., Spurr-Michaud, S.J., Senchyna, M., Ritter 3rd, R., Schaumberg, D., 2011. Comparison of mucin levels at the ocular surface of postmenopausal women with and without a history of dry eye. Cornea 30, 1346-1352.

Hanlon, P., Nicholl, B.I., Jani, B.D., Lee, D., McQueenie, R., Mair, F.S., 2018. Frailty and pre-frailty in middle-aged and older adults and its association with multimorbidity and mortality: a prospective analysis of 493737 UK Biobank participants. Lancet Public Health 3, e323-e332.

Hasko, G., Cronstein, B., 2013. Regulation of inflammation by adenosine. Front. Immunol. 4, 85 .

Kim, K., Kim, H.J., Binas, B., Kang, J.H., Chung, I.Y., 2018. Inflammatory mediators ATP and S100A12 activate the NLRP3 inflammasome to induce MUC5AC production in airway epithelial cells. Biochem. Biophys. Res. Commun. 503, 657-664.

Kojima, T., 2018. Contact lens-associated dry eye disease: recent advances worldwide and in Japan. Invest. Ophthalmol. Vis. Sci. 59, DES102-DES108.

Lachman, M.E., Teshale, S., Agrigoroaei, S., 2015. Midlife as a pivotal period in the life course: balancing growth and decline at the crossroads of youth and old age. Int. J. Behav. Dev. 39, 20-31.

Lehallier, B., Gate, D., Schaum, N., Nanasi, T., Lee, S.E., Yousef, H., Moran Losada, P. Berdnik, D., Keller, A., Verghese, J., Sathyan, S., Franceschi, C., Milman, S., Barzilai, N., Wyss-Coray, T., 2019. Undulating changes in human plasma proteome profiles across the lifespan. Nat. Med. 25, 1843-1850.
Lim, R.R., Wieser, M.E., Ganga, R.R., Barathi, V.A., Lakshminarayanan, R., Mohan, R.R., Hainsworth, D.P., Chaurasia, S.S., 2020. NOD-like receptors in the eye: uncovering its role in diabetic retinopathy. Int. J. Mol. Sci. 21.

Niu, L., Zhang, S., Wu, J., Chen, L., Wang, Y., 2015. Upregulation of NLRP3 inflammasome in the tears and ocular surface of dry eye patients. PloS One 10, e0126277.

Paulsen, F., Jager, K., Worlitzsch, D., Brauer, L., Schulze, U., Schafer, G., Sel, S., 2008. Regulation of MUC16 by inflammatory mediators in ocular surface epithelial cell lines. Ann. Anat. 190, 59-70.

Prince, C.S., Noren Hooten, N., Mode, N.A., Zhang, Y., Ejiogu, N., Becker, K.G., Zonderman, A.B., Evans, M.K., 2019. Frailty in middle age is associated with frailty status and race-specific changes to the transcriptome. Aging 11, 5518-5534.

Ramamoorthy, P., Nichols, J.J., 2008. Mucins in contact lens wear and dry eye conditions. Optom. Vis. Sci. 85, 631-642 official publication of the American Academy of Optometry.

Schiffman, R.M., Christianson, M.D., Jacobsen, G., Hirsch, J.D., Reis, B.L., 2000. Reliability and validity of the Ocular Surface Disease Index. Arch Ophthalmol 118, 615-621.

Schmittgen, T.D., Livak, K.J., 2008. Analyzing real-time PCR data by the comparative C (T) method. Nat. Protoc. 3, 1101-1108.

Stagg, J., Smyth, M.J., 2010. Extracellular adenosine triphosphate and adenosine in cancer. Oncogene 29, 5346-5358.

Stapleton, F., Keay, L., Jalbert, I., Cole, N., 2007. The epidemiology of contact lens related infiltrates. Optom. Vis. Sci. 84, 257-272 official publication of the American Academy of Optometry.

Wolffsohn, J.S., Arita, R., Chalmers, R., Djalilian, A., Dogru, M., Dumbleton, K., Gupta, P. K., Karpecki, P., Lazreg, S., Pult, H., Sullivan, B.D., Tomlinson, A., Tong, L., Villani, E., Yoon, K.C., Jones, L., Craig, J.P., 2017. TFOS DEWS II diagnostic methodology report. Ocul. Surf. 15, 539-574.

Zheng, Q., Ren, Y., Reinach, P.S., Xiao, B., Lu, H., Zhu, Y., Qu, J., Chen, W., 2015. Reactive oxygen species activated NLRP3 inflammasomes initiate inflammation in hyperosmolarity stressed human corneal epithelial cells and environment-induced dry eye patients. Exp. Eye Res. 134, 133-140. 